\title{
SOURCES OF THE X-RAYS BASED ON COMPTON SCATTERING *
}

\author{
V.P. Androsov, E.V. Bulyak, P.I. Gladkikh, I.M. Karnaukhov, A.O. Mytsykov, \\ Yu.N. Telegin, A.A. Shcherbakov, A.Yu. Zelinsky
}

\author{
National Science Center "Kharkov Institute of Physics and Technology”, Kharkov, Ukraine \\ e-mail: gladkikh@kipt.kharkov.ua
}

The principles of the intense X-rays generation by laser beam scattering on a relativistic electron beam are described and description of facilities are assigned to produce the X-rays based on Compton scattering is presented. The possibilities of various types of such facilities are estimated and discussed. The source of the X-rays based on a storage ring with low beam energy is described in details and advantages of the sources of such type are discussed.

The results of calculation and numerical simulation had been carried out for laser electron storage ring N100M that is under developing in NSC KIPT show wide prospects of the accelerator facility of such type.

PACS: 29.20.Dh, 29.27.Bd

\section{INTRODUCTION}

Intensive sources of the short-pulse X-rays are needed in various fields of the science and technology like materials, medicine, chemistry, biology, X-ray lithography and analysis. One of the most promising method producing the short-pulse X-rays is Compton scattering of an intense laser flash on a relativistic electron beam. In present time development of a new generation of facilities using that method called Laser Synchrotron Source (LSS) began in several accelerator laboratories all over the world [1-3]. Radiation produced by LSS has several advantages. There are the easy wavelength tunability, the spectrum and spatial distributions and ad extremum a capability to produce the X-rays using electron beams with relatively low energy, that decreases the cost of a facility essentially.

The idea producing high-energy photons through their scattering on relativistic electrons was proposed as far back as 1951-52 by H. Motz [4] and K. Landecker [5] and was developed by R. Milburn [6], F. Arutyunian and V. Tumanian [7] later on. But abilities of laser and accelerator technique of those time did not allow to consider that proposal from practical point of view due to value of Compton scattering cross section is too small $\left(\sigma_{\mathrm{c}} \sim 6.6^{*} 10^{-29} \mathrm{~m}^{2}\right)$.

The progress made to date in development and producing of lasers with high pulse time stability and large average power opened new prospects for the method. Nowadays three types of the LSS are developed: single pass generation scheme based on interaction of an electron beam delivered from a linear accelerator with an intense laser beam; Laser Electron Storage Ring (LESR) based on interaction of an electron beam stored in a storage ring with a laser beam stored in an optical resonator; multiturn generation scheme using interaction of an electron beam with a laser beam and undulator magnetic field installed at the interaction point (IP) simultaneously.

* This work was supported in part by the NATO Program "Science for Peace" under Grant SfP-977982
The presented paper is devoted to description of capabilities of the method in all three types of LSS. The particular attention will be noticed to the description of the LESR N100M that is under designing in NSC KIPT.

\section{SCATTERING REACTION YIELD}

In the case of the scattering of ultra relativistic particles $a_{01}$ of a cylindrical bunch with radius $R$ by particles $a_{02}$ of an ultra relativistic cylindrical bunch with radius $R$, traveling against this one along $X$ axis with uniform particle density distribution in both bunches the number of scattered particles per second $N$ will be given as following:

$$
N=\frac{\sigma N_{01} N_{02}}{\pi R^{2}} f,
$$

where $\sigma$ is total cross section of the scattering, $N_{01}, N_{02}$ are the number of particles in the both bunches, $f$ is a repetition bunch rate.

If ultra relativistic bunches with Gaussian particle density distributions interact under arbitrary interaction angle $\alpha_{0}$, the number of the scattered particles per second $N$ one can write as following:

$$
N=\frac{1}{2 \pi \sqrt{\sigma_{R 01}^{2}+\sigma_{z 02}^{2}}} \frac{\sigma N_{01} N_{02} f}{\sqrt{\sigma_{x 02}^{2}+\sigma_{R 01}^{2}+\left(\sigma_{l 01}^{2}+\sigma_{l 02}^{2}\right) \tan ^{2}\left(\frac{\alpha_{0}}{2}\right)}},
$$

where $\sigma_{R 01}, \sigma_{x 02}, \sigma_{z 02}$ are transverse sizes of interacting bunches, $\sigma_{l 01}, \sigma_{102}$ are longitudinal sizes of interacting bunches.

\section{COMPTON SCATTERING}

At the interaction of a photon with energy $\varepsilon_{\gamma 0}$ and a relativistic electron with energy $E_{0}$ under an angle $\alpha_{0}$ the photon, scattering on an electron is scattered within an angle $\sim 1 / \gamma$, where $\gamma=E_{0}, m c^{2}, m c^{2}$ is the electron rest energy. The kinematics of Compton scattering are shown in Fig 1. 
Following [8,9], let us write basic formulas characterizing Compton scattering.

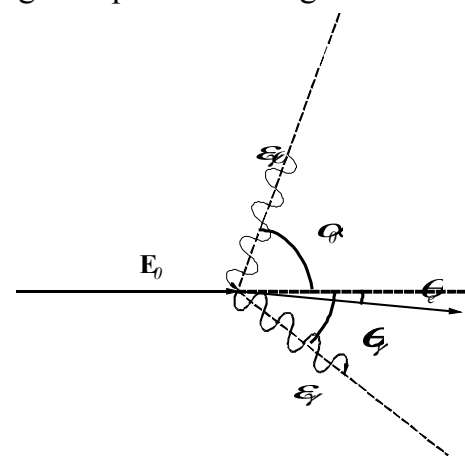

Fig. 1. The kinematics of Compton scattering

Scattered photon energy $\varepsilon_{\gamma}$ depends on scattering angle $\theta_{\gamma}$ as following:

$$
\varepsilon_{\gamma}=\frac{\varepsilon_{\gamma m}}{1+\left(\theta_{\gamma} / \theta_{0}\right)^{2}},
$$

where $\varepsilon_{\gamma m}=\frac{x}{x+1} E_{0}$ is maximum scattered photon energy, $\theta_{0}=\frac{m c^{2}}{E_{0}} \sqrt{x+1}$,

$$
x=\frac{4 E_{0} \varepsilon \gamma_{0} \cos ^{2}\left(\alpha_{0} / 2\right)}{m^{2} c^{4}} \approx 1.53 * 10^{-5} E_{0}[\mathrm{MeV}] \varepsilon_{\gamma_{0}}[\mathrm{eV}] .
$$

Introducing parameter $y=\varepsilon_{\gamma} / E_{0}$ one can write scattering photon angle as a function of its energy in a following form:

$$
\theta_{\gamma}(y)=\theta_{0} \sqrt{\frac{y_{m}}{y}-1},
$$

where $y_{m}=\varepsilon_{\gamma_{m}} / E_{0}$,

Then scattering electron angle is:

$$
\theta_{e}(y)=\theta_{\gamma}(y) \frac{y}{1-y}
$$

In a case of a small colliding angle $\alpha_{0}<<1$ and at meeting a condition $4 \gamma^{2}<<\frac{E_{0}}{\varepsilon_{\gamma 0}}$, which means that scattered photon energy is much less then initial energy of the electron beam we will have:

$$
\varepsilon_{\gamma m} \approx 4 \gamma^{2} \varepsilon_{\gamma} 0 \text {. }
$$

Then

$$
\begin{aligned}
& y_{m}=4 \gamma^{2} \varepsilon_{\gamma 0}, \\
& \theta_{0}=\frac{1}{\gamma} \sqrt{4 \gamma \frac{\varepsilon \gamma 0}{m c^{2}}+1}=\frac{1}{\gamma}, \\
& \varepsilon_{\gamma}=\frac{4 \gamma^{2} \varepsilon_{\gamma 0}}{1+\theta_{\gamma}^{2} \gamma^{2}}=\frac{4 \varepsilon_{\gamma 0}}{\frac{1}{\gamma^{2}}+\theta_{\gamma}^{2}} .
\end{aligned}
$$

The maximum scattered photon energy $\varepsilon_{\gamma_{m}}$ versus electron beam energy $E_{0}$ within energy range from 10 $\mathrm{MeV}$ to $250 \mathrm{MeV}$ for laser with photon energy $\varepsilon_{\gamma 0}=1.17$ $\mathrm{eV}$ (Nd:YAG laser) is shown in Fig. 2. It is clear that it is possible to produce photon beam with photon energy in an energy range from several $\mathrm{keV}$ up to several $\mathrm{MeV}$. It covers up all needs of all known nowadays science and

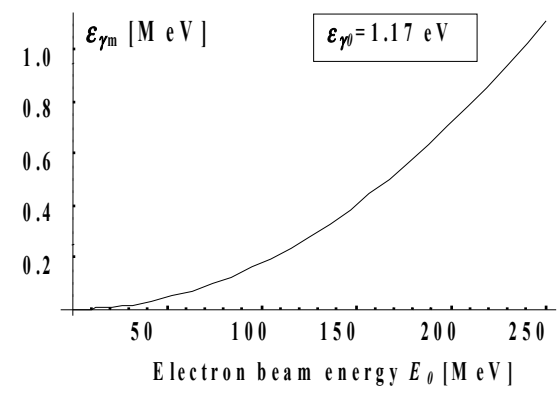

Fig. 2. The maximum scattered photon energy

technological applications of the X-rays. For example, for electron beam energy $100 \mathrm{MeV}$ the maximum photon energy will be of $178 \mathrm{keV}$, and for electron energy $50 \mathrm{MeV}$ will be equal to $45 \mathrm{keV}$.

Electron and photon scattering angles versus scattered photon for initial photon beam with an energy $\varepsilon_{\gamma 0}=1.17 \mathrm{eV}$ and various electron beam energies are shown in Fig. 3.

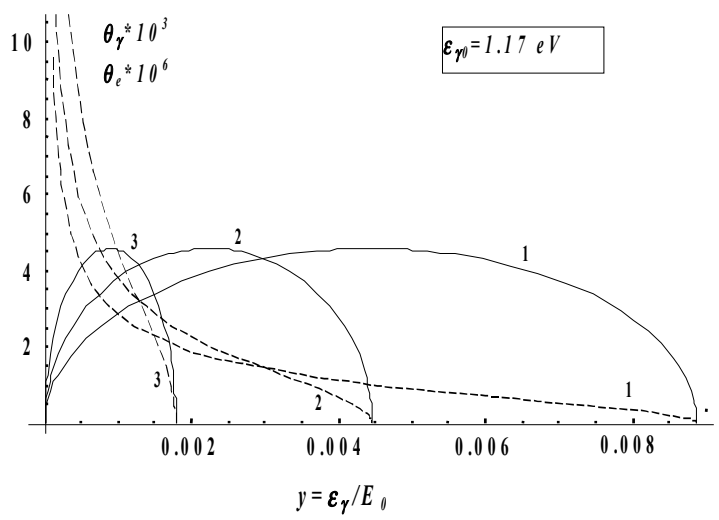

Fig. 3. Electron and photon scattering angles: $1-E_{0}=500 \mathrm{MeV}, 2-E_{0}=250 \mathrm{MeV}, 3-E_{0}=100 \mathrm{MeV}$. Curves: - - photons, - electrons

Differential cross-section of Compton scattering had been given in $[8,9]$. Normalized energy spectrum of scattered photons given by differential cross section and (3) for electron beam with energy $100 \mathrm{MeV}$ and Nd:YAG laser is shown in Fig. 4.

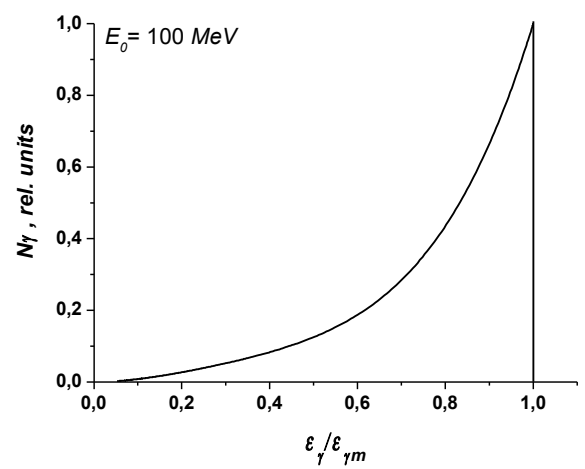

Fig. 4. Normalized energy spectrum of scattered photons

The spectral brightness $B_{\square}$ [photons $/ \mathrm{s} / 0.1 \% / \mathrm{mm}^{2} /$ $\left.\mathrm{mrad}^{2}\right]$ is determined as a number of photons radiated 
within unit solid angle from unit of a source aria in bandwidth equal to $0.1 \%$ of radiation spectrum per second.

During Compton scattering photons are scattered in solid angle $\Delta \Omega=\pi\left(\sigma_{e}^{2}+\sigma_{\gamma}^{2}\right)$, where $\sigma_{e}^{\prime}$ is electron beam divergence, $\sigma_{\gamma}^{\prime} \approx \frac{1}{\gamma}$ is photon beam divergence.

Thus, Compton scattering spectral brightness is determined by intensity of the scattering, energy spectrum curve (Fig. 4) and geometrical parameters of the interacted bunches.

Thus we can formulate the main properties of the Xrays generated by Compton scattering of photons on relativistic electrons:

1. Compton scattering allows to produce the X-rays using photon source with low photon energy and electron beam with low energy too. It is possible to change the X-rays energy, changing electron beam energy.

2. Energy end angle distributions of the produced Xrays allow to get a high level of the X-rays monochromaticity merely by beam collimation and to decrease background radiation essentially without use of a monochromator.

3. The energy spectrum of produced photons lies from several $k e V$ up to several $M e V$. Such radiation is very attractive for all X-rays applications beginning from the solid state physics, the X-rays lithography, spectroscopy, biology and medicine and ending with the nuclei physics and particle physics.

\section{COMPTON SCATTERING FACILITIES}

In consequence of progress in construction of powerful lasers with high repetition rate in the middle of the $90^{\text {th }}$ years the interest in development and design of intensive X-rays sources based on Compton scattering (LSS) was renovated in many accelerators laboratories all over the world. Nowadays three types of LSS are under consideration:

1. Single-pass facility - source is based on interaction of an electron beam from a linear accelerator with a powerful laser pulse. In such facility, the multi-bunch electron beams are delivered by the linac or microtron. At present time photoinjectors allow to receive electron beams with intensity $N_{e} /$ bunch of about $10^{10}$, bunch duration equal to a few $p s$, pulse repetition rate up to $1000 \mathrm{~Hz}$ and with several thousand bunches per pulse. Thus, the average electron beam current can be of about few $m A$. Normalized emittance of such accelerator can be of about $1 \mathrm{~mm}$ mrad. The laser pulse repetition rate in such facility has to be chosen in such way to adjust repetition rate of electron bunches.

Let us estimate the photon flux intensity and spectral brightness for the linear accelerator with parameters based on Brazil, Sao Jose dos Campos linear accelerator for neutron production and research [10]. Parameters of the linac and the laser optical system are shown below.

\section{Linear accelerator:}

Electron beam energy, $\mathrm{MeV}$................. 100

Electrons per bunch ........................... $4 * 10^{9}$

Normalized RMS emittance, $\mathrm{mm}$ mrad 1

Bunch repetition rate, $\mathrm{Hz}$................. $\quad 3 * 10^{9}$

Linac repetition rate, $\mathrm{Hz}$.................. 1000

Average current, $m A$......................... 2

Laser-optical system:

Photons energy, $\mathrm{eV}$............................ 1,17

Average laser power, $W \ldots \ldots \ldots \ldots \ldots \ldots \ldots . . .100$

Spot radius, $\mu \mathrm{m}$................................. 50

Photons per pulse in an optical

resonator....

$5.335^{*} 10^{16}$

Flash energy, $J$................................... 0.01

Then according to (1) the X-rays intensity will be:

$N_{\gamma}[\mathrm{phot} / \mathrm{s}]=2.4 * 10^{11}$

And spectral brightness is equal to:

$B_{\gamma}\left[\mathrm{phot} / \mathrm{s} / 0.1 \% \mathrm{bw} / \mathrm{mm}^{2} / \mathrm{mrad}^{2}\right]=2.16^{*} 10^{9}$.

Facilities of such type are used for producing of the femtosecond X-rays [1, 11], and construction single pass LSS for medical application is planned too [12].

2. Intracavity scheme LESR - electron beam circulating in a storage ring interacts with stored in an optical resonator laser pulse. Resonator is formed with high reflectivity mirrors. The optical length of a laser pulse in the resonator is chosen in such way to adjust with circulation frequency of the electron beam in the storage ring. So, electrons will interact with laser pulse at optical resonator focus on every turn. The possibility to provide much more value of the average electron current is an obvious advantage of such scheme in compare with previous one. Let us estimate the X-rays intensity and spectral brightness for the following parameters of a storage ring and a laser optical system:

\section{Storage ring:}

Electron beam energy, $\mathrm{MeV} \ldots \quad 100$

Electrons per bunch ............... 2.08*10 $10^{10}$

RMS emittance, $m m$ mrad ..... $10^{-2}$

Circumference, $m$................ 10

Bunch repetition rate, $\mathrm{Hz} \ldots \ldots . . \quad 3 * 10^{7}$

Average current, $m A$............. 100

Laser-optical system:

Photons energy, $\mathrm{eV} \ldots \ldots \ldots \ldots \ldots . . . . . \quad 1,17$

Average laser power, $W \ldots \ldots \ldots . \quad 100$

Spot radius, $\mu m$................... 50

Photons per pulse in an

optical resonator

\section{$5.335^{*} 10^{16}$}

Flash energy, $J$.................... 0.01

Then according to (1) the X-rays intensity will be:

$N_{\gamma}[$ phot $/ s]=2.81 * 10^{14}$

And spectral brightness is:

$B_{\gamma}\left[\mathrm{phot} / \mathrm{s} / 0.1 \% \mathrm{bw} / \mathrm{mm}^{2} / \mathrm{mrad}^{2}\right]=4.1 * 10^{12}$.

Nowadays a possibility of LESR engineering is considered in several accelerator laboratories $[3,12,13]$.

3. Intracavity scheme incorporated with FEL - an electron beam of a storage ring generates coherent laser beam passing along an undulator and then scatters that laser beam trough Compton scattering. When electron and photon beams travel in the same direction the 
electron beam passing along the undulator generates photons with the same energy as stored in the optical resonator. Then photon beam is reflected from a mirror and meet the electron beam generating hard X-rays trough Compton scattering. An optical resonator length is chosen to synchronize electron and photons beams. That solution is the most complicate and expensive although taking into account spectral brightness which one can get with FEL (up to $10^{22} \mathrm{phot} / 0.1 \mathrm{bw} / \mathrm{s} / \mathrm{mm}^{2} / \mathrm{mrad}^{2}$ ), it seems very attractive from the generation of the extremely high intensive X-rays point of view. In such way radiation with spectral brightness up to $10^{20}$ phot $/ 0.1 \mathrm{bw} / \mathrm{s} / \mathrm{mm}^{2} / \mathrm{mrad}^{2}$ can be produced. Despite the promising prospects this scheme has very serious problems for realization. The necessary condition for radiation generation in FEL is quasimonochromaticity, in other words it is necessary to provide such small energy spread as it possible. Simultaneously, during Compton scattering energy spread in the electron beam under condition of such intensive radiation can reach up to several percents that leads to generation condition breaking down.

Despite obvious difficulties in realization of the facilities of such type, project design is carrying out in several places such as Duke university [14] and at the UVSOR FEL [15].

\section{COMPACT LESR DESIGN}

Despite obvious LESR advantages of other LSS types for the X-rays intensity over LSS based on linac scheme and for low cost and realization simplicity over LSS based on FEL using development of a compact LESR is very complicate task. To provide stable electron beam motion during a long time under the condition of interaction with intense laser beam it is necessary to solve the following problems:

1. One has to ensure the ring energy acceptance of about $5 \ldots 10 \%$ in order to provide the reasonable beam quantum lifetime. It needs the RF-voltage of several MV. There is no place to accommodate a large number of RF-cavities in a compact storage ring.

2. Under conditions of a large energy spread the electron dynamics is stipulated not only by the first order effects but high order effects on momentum deviation. Hence, to focus electron beam at IP properly one has to suppress the second order effects at this azimuth. Moreover, the chromatic effects can results in beam diffusion on synchrobetatron resonances for high RF-voltages that lead to particles losses.

3 . It is necessary to provide the maximum electron beam density at the IP. This is achieved by decreasing the values of radial and vertical amplitude functions at the IP.

4. At increasing electron beam density the effect of intrabeam scattering (IBS) on transverse beam sizes is increased drastically.

5. The necessity to provide strong electron beam focusing at the IP in a compact storage ring with one achromatic straight section reduces the order of storage ring symmetry down to $N=1$, and it drastically extends the spectrum of azimuthal harmonics of nonlinear perturbations. Simultaneously a strong focusing of the beam results in a high natural chromaticity of the ring, the compensation of which requires the installation of strong sextupole lenses. It restricts the dynamic aperture (DA) of the ring.

Let us consider some of these problems in more details.

In a cyclic accelerator the reference orbit deviation in orbital plane $\Delta x_{C}$ is given by:

$$
\Delta x_{C}=\eta_{1} \delta+\eta_{2} \delta^{2}+\ldots
$$

where $\eta_{1}, \eta_{2}$ are linear and quadratic dispersion functions, which one can write as following:

$$
\begin{gathered}
\eta_{1}(s)=\frac{1}{2 \sin \pi Q_{x}} \int_{s}^{s+C} \frac{\sqrt{\beta_{x}(s) \beta_{x}(\sigma)}}{\rho(\sigma)} \cos \left(\pi Q_{x}-\mu_{\sigma s}\right) d \sigma \\
\eta_{2}(s)=-\eta_{1}(s)+\frac{1}{2 \sin \pi Q_{x}} * \int_{s}^{s+C} \sqrt{\beta_{x}(s) \beta_{x}(\sigma)} \cos \left(\pi Q_{x}-\mu_{\sigma}\right) * \\
\left(K_{1}(\sigma) \eta_{1}(\sigma)-\frac{1}{2} K_{2}(\sigma) \eta_{1}^{2}(\sigma)\right) d \sigma,
\end{gathered}
$$

where $K_{1}, K_{2}$ are quadrupole and sextupole strengths, $Q_{x}$ and $\mu_{\sigma_{s}}$ are tune and phase advance of the horizontal betatron oscillations, $C$ is a storage ring circumference. Thus, dispersion at the IP results in horizontal beam size growing due to synchrotron oscillations. When the $\delta$ is small it is enough to take into account linear components in momentum deviation only.

In LESR one has to use bending magnets with large bending angle for facility compactness. Hence, the dispersion $\eta_{1}$ is large. Besides, the requirement to produce small beam size at the IP leads to use strong quadrupoles. It results in high chromaticity value. For chromaticity compensation strong sextupoles are needed. Then, as it follows from (11), quadratic dispersion can be large too. Hence, the IP has to be achromatic both in the first and in the second order. In the opposite case Compton scattering intensity will be very low due to large electron beam size.

The first order achromaticity is provided with linear lattice magnetic elements only (bending magnets and quadrupoles). To achieve the second order achromaticity along with proper choice of sextupoles strength the determined sextupoles phasing at sections with nonzero dispersion is needed.

Focusing properties of the magnetic elements depend on particle energy. It determines betatron tunes and amplitude functions dependence on particle energy. Quantitatively these dependences is characterized by derivatives $\partial Q_{x, y} / \partial \delta, \partial{ }^{2} Q_{x, y} / \partial \delta^{2}, \ldots, \partial \beta_{x, y} / \partial \delta, \ldots$ Amplitude functions and betatron tunes changes determines the nonlinear nature in particle betatron motion. It leads to growth of effective emittances $\varepsilon_{x, y}$ and decreases a facility brightness.

The quadratic components in particle transverse displacement in terms of $\delta$ (10) result in quadratic effects in orbit length depending on particle energy. The momentum compaction factor $\alpha$ becomes function of energy 
$\alpha=\alpha(\delta)=\alpha_{1}+\alpha_{2} \delta$

where

$$
\alpha_{1}=\frac{1}{C_{0}} \int \frac{\eta_{1}}{\rho} d s, \quad \alpha_{2}=\frac{1}{C_{0}} \int\left(\frac{\eta_{2}}{\rho}+\frac{\eta_{1}^{\prime 2}}{2}\right) d s
$$

are linear and quadratic momentum compaction factor, $\eta_{1}^{\prime}=d \eta_{1} / d s$

In a linear approximation in terms of $\delta$ energy acceptance of a storage ring is the larger the smaller linear momentum compaction is:

$\sigma_{\mathrm{RF}} \propto \alpha_{1}^{-1 / 2}$.

Consideration of the quadratic components shows [16] that beginning from certain $\alpha_{l}$ critical value the RF acceptance value begins to decrease. The limited value of RF acceptance is given by the following expression:

$\sigma_{R F \max }=\left|\alpha_{1} / \alpha_{2}\right|$

Under large quadratic effects $\left(\eta_{2}\right.$ - dominated beam) the RF acceptance shape is distorted. An example of such one is depicted in Fig. 5.

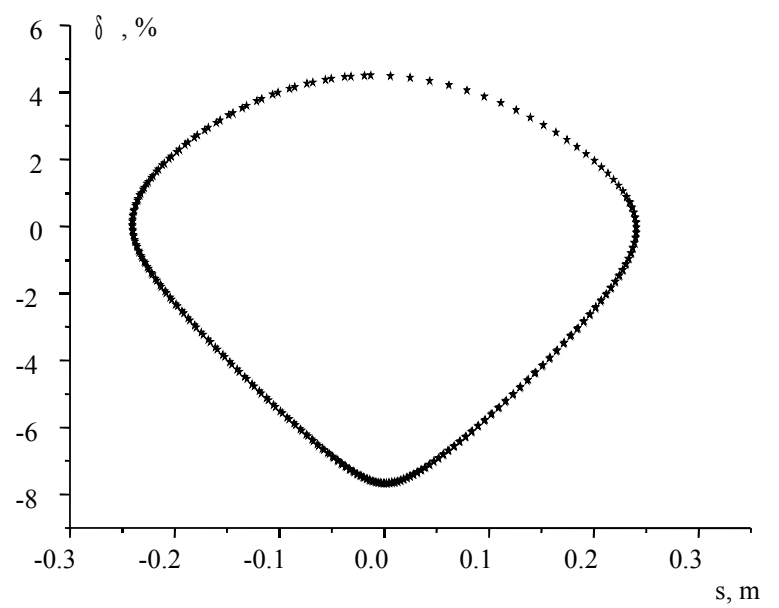

Fig. 5. The RF acceptance shape under effect of large quadratic effects

Choosing a storage ring lattice it has to be taken into account. One must not to design lattice with a large quadratic dispersion where providing of necessary value of the RF acceptance is impossible.

Considering the all above we had developed a concept of a compact LESR with controlled momentum compaction factor $[3,17]$. The base idea of the concept is decreasing of a storage ring momentum compaction factor. It leads to decreasing of a bunch length and increases RF acceptance (13). Thus it gives an opportunity to keep a necessary value of the RF acceptance with much lower value of RF voltage.

An example of a lattice which is both as quite simple as allowing to control the momentum compaction factor and meeting the all above-mentioned requirements is depicted in Fig. 6.

The lattice consists of two bending arcs BM1-BM2 and BM3-BM4 with a short straight section each and two long (matching) straight section BM1-BM4 and BM3-BM2. There are on two quadrupole magnets at each short section. It allows to meet focusing condition and to control the momentum compaction. Bending magnets is used in the arcs have a combined focusing function. On two objectives with three or four lenses are placed at each long straight section for electron beam focusing. The long straight sections BM1-BM4 and BM3-BM2 are achromatic when the storage ring works in a normal mode with large momentum compaction (NM). In a mode with a low momentum compaction (LA) this one is changed due to appearance of the dispersion with an opposite sign in the arc bending magnet and straight section BM3-BM2 becomes chromatic as it shown in Fig. 7.

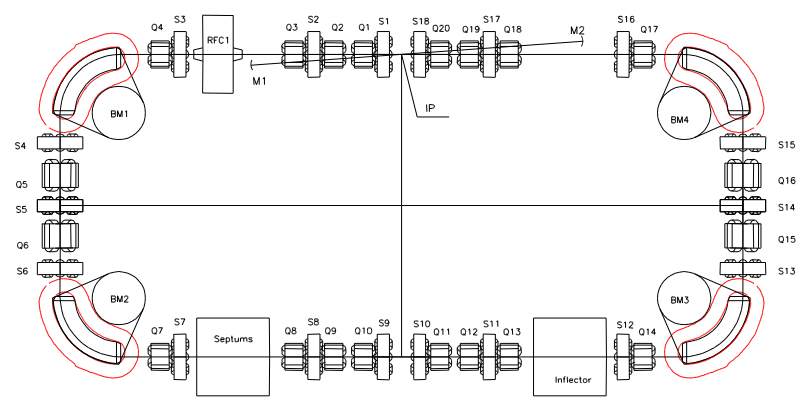

Fig. 6. The layout of the LESR with controlled momentum compaction factor N100M. BM - bending magnets, $Q, S$ - quadrupole and sextupole magnets, $R F C-R F$-cavity, $M$ - optical resonator mirrors

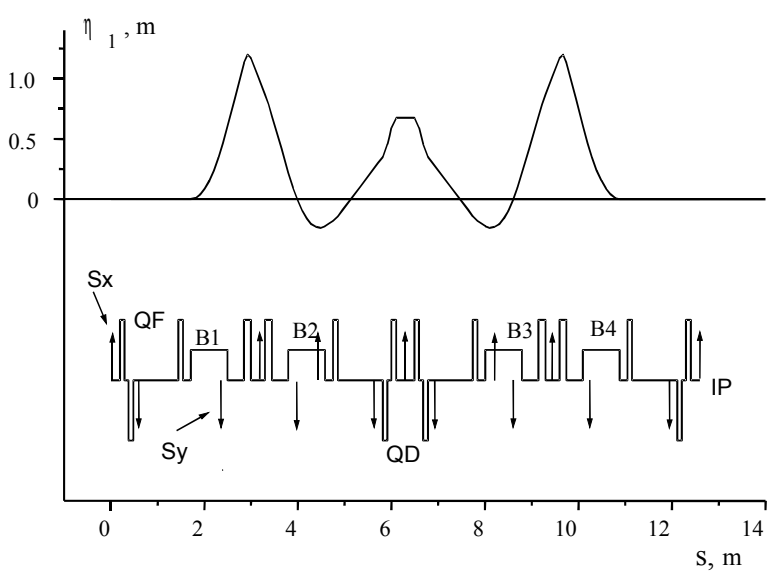

Fig. 7. The dispersion function and the distribution of sextupoles for the correction of the chromatic effects. $S_{x}, S_{y}$ are sextupoles, B1-B4 are bending magnets, $Q F$ and $Q D$ are quadrupoles

In addition to RF acceptance support a long straight section with non-zero dispersion allows to compensate chromatic effects at the IP. To correct the chromatic effects sextupole lenses are placed along the orbit at the azimuths with non-zero values of the dispersion function. The lattice is designed to provide the required phase advances of betatron oscillations between sextupoles and to compensate the second order dispersion and the dependence of radial and vertical amplitude functions on particle energy at the IP. Achromatic long straight section sextupoles correct the DA value.

Calculations have shown that the above described principles of a LESR designing allow to develop the Xrays source with the ring circumference of about $10 \mathrm{~m}$ using traditional technologies of magnetic elements manufacture. 
Using these principles as a background, a lattice of LESR N100M for NSC KIPT with beam energy up to $225 \mathrm{MeV}$ had been designed (Fig. 6). Four $90^{\circ}$-bending magnets with field index equal to $0.6,20$ quadrupole lenses (on 2 in each short straight section and on two 4lenses objectives in each long straight section), 18 sextupole lenses (on 3 in each short straight section and on 6 in each long straight section) are used in the lattice. Circumference of the ring is $15.4176 \mathrm{~m}$. Betatron oscillations tunes are $Q_{x}=3.15, Q_{z}=2.21$. Electron beam sizes in linear approximation for electron energy 43 $\mathrm{MeV}$ and coupling coefficient equal to 0.6 are $\sigma_{x}=\sigma$ $z=20 \square \mathrm{m}$. The lattice allows decreasing the momentum compaction factor down to $10^{-2}$, providing $\mathrm{RF}$ acceptance of about $4 \%$ with a small RF voltage. The $\mathrm{DA}$ at the IP is equal to $\pm 3 \mathrm{~mm}$. Calculated with MAD code amplitude functions and phase trajectories of a particle at IP is shown in Fig. 8-9. As it is clear from Fig. 9 the chromatic effects are suppressed in the lattice.

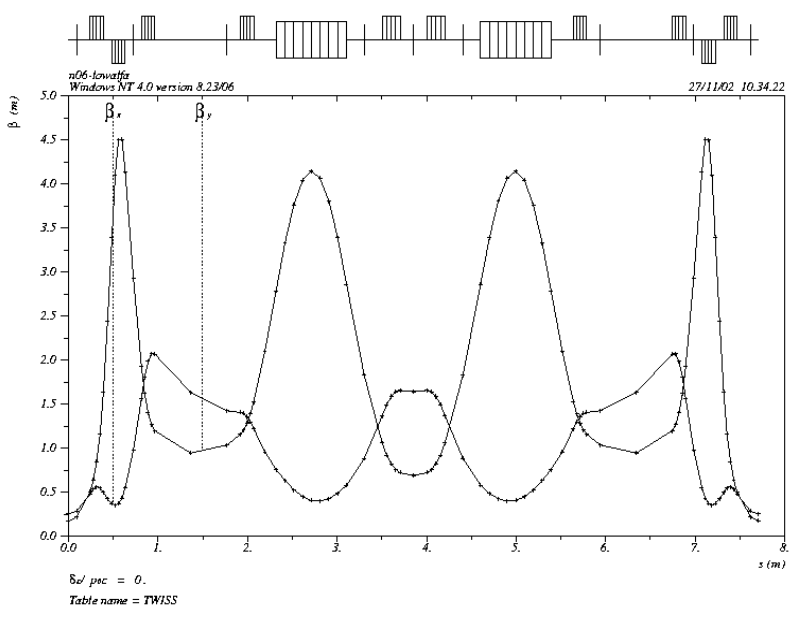

Fig. 8. Amplitude functions of LESR N100M (output from $M A D$ code)

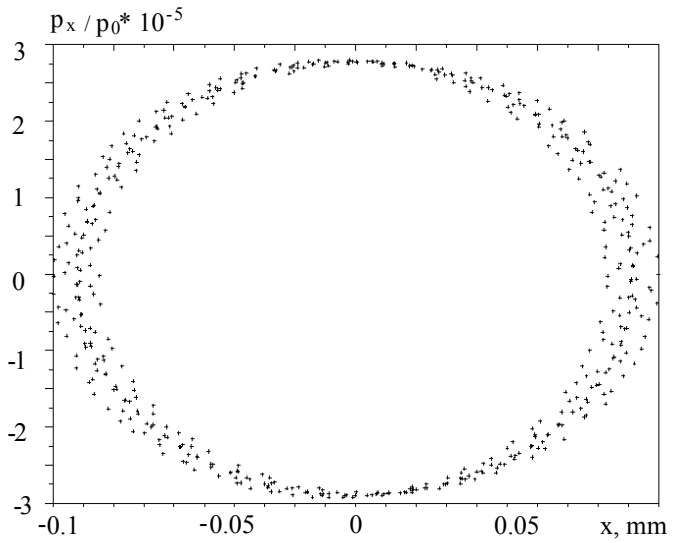

Fig. 9. Phase trajectories of a particle at IP in LM. Initial particle coordinates: $x=100 \mu, x^{\prime}=0, y=100 \mu$, $y^{\prime}=0, s=0, \delta=1 \%$

As it was already noted the IBS will effect essentially on the electron beam dynamics due to low beam energy in the LESR. It is very difficult task to consider this effect correctly with analytical means for it is self-congruent task. It is especially difficult for nonzero electron-photon interaction angle. In this case efficiency of Compton scattering depends on longitudinal electron beam size that, in its turn, is determined by Compton scattering intensity. To consider the IBS we offered the following algorithm. Using [18] we calculate a table of an emittance growth rates in a range transverse and longitudinal emitance values which are determined by joint effect of the IBS and Compton scattering. During the simulations the growth rates are being correcting according to the real distribution of the electron beam. The time between two sequential corrections has to be much less then growth rates. Meeting the condition we get good matching of simulation results for steady state beam emittances and estimations were made with [18]. Calculations had been carried out show that for the above mentioned lattice and electron beam parameters and without taking into account laser-electron interaction beam sizes are grown up to $\square_{\mathrm{x}}=\square_{\mathrm{z}}=200 \mathrm{Zm}$. But we would have to expect decreasing of the steady state beam size due to Compton scattering.

Let us estimate intensity of the generated by LESR N100M the X-rays beam for the laser optical system (LOS) with the following parameters:

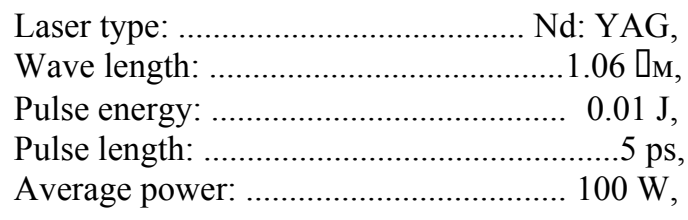

At such parameters of LOS the number of particles in a laser flash will be $N_{p h}=5.33 \cdot 10^{16}$. For the beam current $I=0.01 \mathrm{~A}$ the number of electron per bunch will be $N_{e}=3.1 \cdot 10^{9}$. Assuming Gaussian distributed electron beam with steady state size equal to $50 \square \mathrm{m}$ in the horizontal plane, $25 \square \mathrm{m}$ in the vertical plane and $15 \mathrm{~mm}$ in the longitudinal direction interacts with a Gaussian distributed photon beam with a radius equal to $50 \mathrm{Dm}$ at the IP and $15 \mathrm{~mm}$ longitudinal size according (2) the Xrays intensity will be:

$N_{\square}[\mathrm{phot} / \mathrm{s}]=1.7 \cdot 10^{12}$.

Taking into account that photon beam divergence $\sigma_{\gamma}^{\prime} \approx 5 \cdot 10^{-3} \mathrm{rad}$ and electron beam divergence $\sigma_{e}^{\prime}=5 \cdot 10^{-4} \mathrm{rad}$ spectral brightness will be:

\section{$B_{\square-}\left[\mathrm{phot} / 0.1 \% / \mathrm{bw} / \mathrm{s} / \mathrm{mm}^{2} / \mathrm{mrad}\right]=2.17 \cdot 10^{10}$.}

For the investigations of the electron beam dynamics in a LESR taking into account electron photon interaction we had developed the proper algorithms and codes [19] based on the Monte-Carlo Method. The developed code allows us to carry out full-scale 3D simulation of the electron beam motion in the LESR through the period of time that exceeds the real damping times (millions of turns). The results of simulation for the lattice LESR N100M (Fig. 6) with the above 
mentioned LOS parameters are shown in Fig. 10, 11. Simulations carried out for the beams with Gaussian density distribution and under interaction angle equal to $\square_{0}=0.06$ with momentum compaction factor $\square=0.02$. One can see from the figures that results of simulations in order the X-rays intensity are in a good agreement with our estimations we made with (2).

\section{LASER OPTICAL SYSTEM OF LESR N100M}

The essential feature of the X-ray source based on Compton scattering is an optical cavity (OC) in which a laser pulse is stored for interaction with the electron beam. The development of the efficient and, at the same time, the moderate-cost laser-optical system (LOS) is the crucial task of this project.

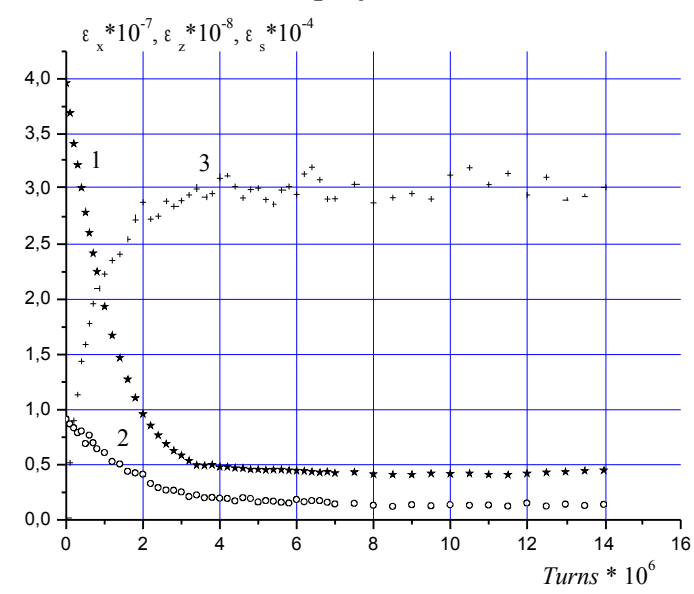

Fig. 10. Transverse (1,2) and longitudinal (3) emittances at Compton scattering in LM

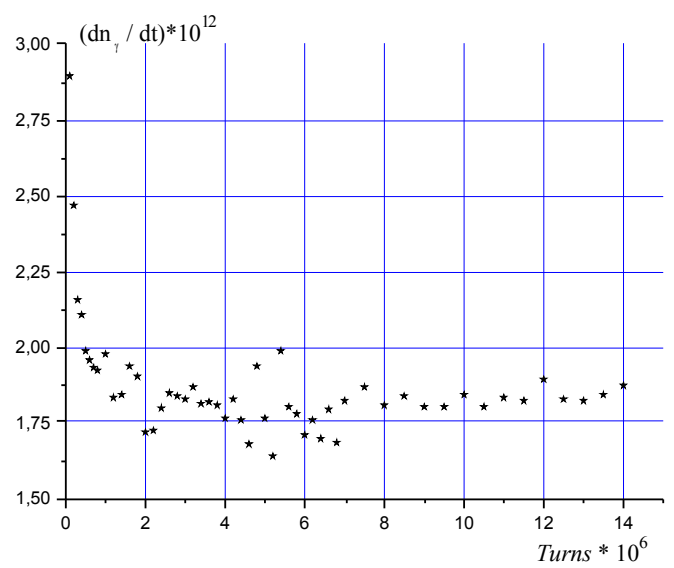

Fig. 11. Intensity of Compton radiation in $L M$

To obtain the required X-ray beam intensity we intend to store in $\mathrm{OC}$ the laser pulse with the parameters: stored pulse energy of $10 \mathrm{~mJ}$, pulse duration of $\sim 50 \mathrm{ps}$ and pulse repetition rate of $60 \mathrm{MHz}$. The estimates show that for direct excitation of the OC, formed by two high-reflectivity spherical mirrors, (see Fig. 12a) one needs a high-power $\mathrm{Nd}$ laser. The required laser power $P_{l}$ is presented in the second line of Table 1 versus mirror reflectivity $r$. One can see that this approach can be realized only for OC mirrors with $r \geq$ 0.9999 . Such a system is unique concerning both the laser (pulse power of $\sim 20 \mathrm{~kW} /$ pulse, repetition rate of $60 \mathrm{MHz}$ ) and the mirrors ( $\mathrm{r} \sim 0.9999)$.

Nevertheless, it is supposed to use such a scheme for the medical X-ray source at KEK [20].

To eliminate the necessity to use a high-frequency laser one can apply the LOS scheme presented in Fig. 12b. It incorporates a low-power $\mathrm{Nd}$ laser that forms the trigger pulse of the required duration and a 3mirror optical cavity (in principle, this ring cavity can have a different configuration) with a laser amplifier placed in one of the cavity arms. This scheme allows multiplying the initial laser pulse to obtain the required pulse repetition rate and, at the same time, maintaining the required pulse power. The parameters of such a system incorporating the 3-mirror traveling wave cavity (TWC) with reflectivity $r_{l}=0.1$ and $r_{2}=r_{3}=0.9$ are listed in the third line of Table 1.

a)

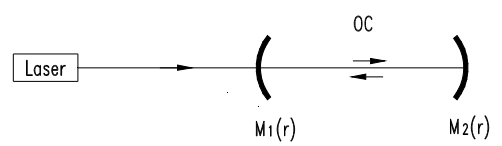

b)

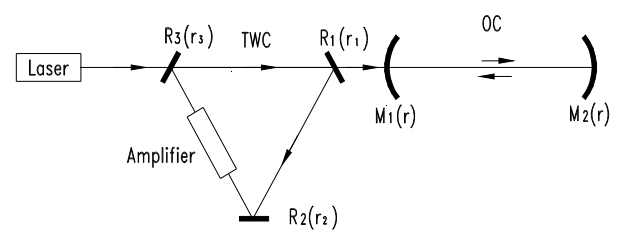

c)

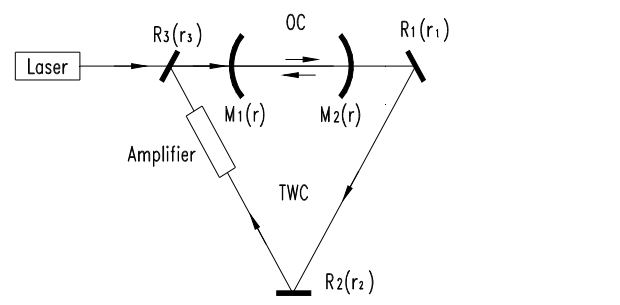

Fig. 12. The LOS for Compton scattering X-ray sources: a) direct excitation of $O C$ with a highfrequency laser; b)LOS with TWC for increasing the laser pulse repetition rate; c) LOS with OC placed in one of TWC arms. Notations: $M_{1}(r), M_{2}(r)$ - OC mirrors with reflectivity $r ; R_{1}\left(r_{1}\right), R_{2}\left(r_{2}\right), R_{3}\left(r_{3}\right)-T W C$ mirrors with corresponding reflectivity

Despite of deterioration of energy characteristics of such a LOS, stipulated by the additional losses in the TWC mirrors, beside the previous one, it has some advantages and it is implemented in DULY project [21]. The possible deterioration of temporal and spatial parameters of the short laser pulse $(\tau<1 \mathrm{ps})$, arising from the dispersion in the resonance system and nonlinear effects in the amplifier, can be reduced by incorporating in the TWC the additional elements (pulse stretcher and compressor). It can result in further deterioration of LOS energy parameters. The requirements to the OC mirror parameters in this scheme are still high $(r \geq$ 0.999).

Considering the high level of energy losses through the TWC mirrors in the above-mentioned scheme we propose to place the OC into the TWC that will produce a continuous wave laser beam at IP. This scheme is presented in Fig. 12c, and its parameters are listed in the 
last line of Table 1. Results are obtained for TWC mirror reflectivity $r_{i}(i=1,2,3)=0.99$.

One can see from the table that this approach allows us to achieve an accumulation of laser photons in the OC with mirrors having relatively moderate parameters (and, consequently, moderate prices). We consider this scheme of LOS preferable for implementing in our project.

Table 1. Laser and amplifier parameters

\begin{tabular}{ccccc}
\hline \hline Reflectivity & 0.99 & 0.999 & 0.9999 \\
\hline Laser power, $k W$ & 6.0 & 0.6 & 0.06 \\
\hline \multirow{2}{*}{$\begin{array}{c}\text { Amplifier } \\
\text { power, } W\end{array}$} & $\mathrm{TWC}$ & 7500 & 750 & 75 \\
\cline { 2 - 5 } & $\mathrm{OC}$ in TWC & 180 & 18 & 1.8 \\
\hline \hline
\end{tabular}

\section{CONCLUSION}

Thus, as a conclusion one can note that LSS is a facility capable to generate the intense X-rays for medicine, science and technological purposes. Among LSS types LESR is the most promising one for the reasons the values of generated radiation intensity and spectral brightness. Moreover, LESR is competitive with synchrotron radiation sources first of all for the cost of facility. Table 2 shows economical characteristics of the biggest synchrotron light sources and LESR N100M.

Table 2. Synchrotron radiation sources and LESR N100M parameters

\begin{tabular}{l|c|c|c|c}
\hline \hline & $\begin{array}{c}\text { Spring } \\
-8\end{array}$ & APS & ESRF & $\begin{array}{c}\text { LESR } \\
\text { N100M }\end{array}$ \\
\hline \multicolumn{1}{c}{ Energy, GeV } & 8 & 7 & 6 & 0.225 \\
\hline X-rays range, $\mathrm{keV}$ & \multicolumn{5}{c}{$30-70$} & & $3-900$ \\
\hline Spectral brightness & & $\sim 10^{14}$ & & $2 \cdot 10^{10}$ \\
\hline Circumference, $m$ & 1436 & 1060 & 776 & 15 \\
\hline Total cost, bill. Euros & 1 & 0.4 & 0.2 & 0.001 \\
\hline Beam lines number & 57 & 69 & 30 & 1 \\
\hline $\begin{array}{l}\text { Cost per channel, } \\
\text { mill. Euros }\end{array}$ & 16 & 7 & 7 & 1 \\
\hline \hline
\end{tabular}

The investigations carried out with the proposed low- $\alpha$ schemes of the LESR with compensation of chromatic aberrations at the IP proved a possibility to construct the sources of the intensive X-ray beams based on compact storage rings.

\section{REFERENCES}

1. S. Kashiwagi, Y. Hama, H. Ishikawa et al. Experiment of $X$-ray generations using inverse Compton scattering at Waseda University. Proc. of EPAC 2002, 2002, v. 1, p. 694-697.

2. K. Nakajima, H. Nakanishi, A. Ogata, F.Q. Zhang. Proposal of a compact high brightness laser synchrotron light source for medical applications. Proc. of PAC 97, 1997, p. 2371-2373. 3. E. Bulyak, P. Gladkikh, A. Zelinsky et al. Compact X-ray source based on Compton scattering // Nucl. Inst. \& Methods. 2002, A, № 487, p. 241-248.

4. H. Motz. Applications of the radiation from fast electron beams // Journal of Apply Physics. 1951, v. 22, № 527, p. 527-531.
5. K. Landecker. Possibility of frequency Multiplication and wave amplification by means of some relativistic effects // Physical Review. 1952, v. 86, №6, p. 852-855.

6. R.H. Milburn. Electron Scattering by an Intense Polarized Photon Field // Phys. Rev. Lett. 1963, № 10, p. 75-78.

7. F.R. Arutyunian, V.A. Tumanian. Compton effect on relativistic electrons and the possibility of obtaining beams of hard D-quanta // JETP, 1963, v. 44 , p. $2100-2103$.

8. V. Telnov. Principles of photon colliders // Nucl. Inst. \& Methods. 1995, A, № 355, p. 3-18.

9. I. Ginzgurg, G. Kotkin, V. Serbo, V. Telnov. Colliding $\gamma$ e and $\gamma \gamma$ beams based on the single-pass $\mathrm{e}^{+} \mathrm{e}^{-}$colliders (VLEPP type) // Nucl. Inst. \& Methods. 1983, A, № 205, p. 47-69.

10. J. Clendenin, L Rinalfi, K. Takata, D. Warner. Compendium of scientific Linacs: CERN 96-32, p. 10.

11. K.J. Kim, S. Chattopadhyay, C.V. Shank. Generation of femtosecond X-rays by $90^{\circ}$ Thompson scattering // Nucl. Inst. \& Methods. 1994, A, № 341, p. 351-354.

12. K. Nakajima, H. Nakanishi, A. Ogata et al. Proposal of a compact high brightness laser synchrotron light source for medical applications. Proc. of PAC 97, 1997, p. 2371-2373.

13. Zh. Huang. Generation of intense X-rays and low-emittance electron beams in a laserelectron storage ring. Proc. of the 2nd ICFA Advanced Accelerator Workshop on the Physics of High Brightness Beams, 2000, p. 21-32

14. T. Scott et al. The TUNL-Fell inverse Compton D-ray source as a nuclear physics facility // Nucl. Inst. \& Methods. 1996, A, № 378, p. $1-20$.

15. M. Hosaka, H. Hama, K. Kimura et al. Observation of intracavity Compton backscattering of the UVSOR free electron laser // Nucl. Inst. \& Methods. 1997, A, № 393, p. 525-529.

16. L. Lin, C. E. T. G. da Silva. Second order single particle dynamics in quasi-isochronous storage rings and its application to the LNLS-UVX ring // Nucl. Inst. \& Methods. 1993, A, № 329, p. $9-15$

17. P. Gladkikh, I. Karnaukhov, A. Zelinsky. Inten-se X-ray sources based on Compton scattering in laser electron storage ring // VANT. 2002, № 2, p. 72-75.

18. J. Bjorken, S.K. Mtingwa. Intrabeam scattering // Particle Accelerators. 1983, № 13, p. 115-119.

19. P. Gladkikh, I. Karnaukhov, Yu. Telegin, A. Zelinsky. The electron beam dynamics simulation in the laser electron storage ring involving Compton and intrabeam scattering // VANT. 2002, № 2, p. 78-81.

20. J. Urakawa at al. Laser Super Cavity. Proc. of 21-th ICFA beam dynamics workshop on LaserBeam Interactions, 2001, Paper TH2- 
21. D. Yu, B. Stuart. A laser pulse trapper for Compton backscattering applications. Proc. of PAC-97, 1997, p. 886-888. 